\title{
Advanced signal processing tools for helicopters' future Health and Usage Monitoring Systems (HUMS)
}

\author{
Alexandre Mauricio ${ }^{1,2}$, Linghao Zhou ${ }^{3}$, Mathew Greaves ${ }^{4}$, Wenyi Wang ${ }^{5}$, David $\mathrm{Mba}^{6}$, and \\ Konstantinos Gryllias ${ }^{1,2, *}$ \\ ${ }^{1}$ Department of Mechanical Engineering, KU Leuven, 3001, Leuven, BE \\ ${ }^{2}$ Dynamics of Mechanical and Mechatronic Systems, Flanders Make, 3001, Leuven, BE \\ ${ }^{3}$ School of Engineering, London South Bank University, SE1 0AA, London, UK \\ ${ }^{4}$ HeliOffshore, UK \\ ${ }^{5}$ Air Vehicles Division, Defence Science and Techn. Organization (DSTO), Melbourne, VIC 3011, AU \\ ${ }^{6}$ Faculty of Technology, De Montfort University, LE1 9BH, Leicester, UK
}

\begin{abstract}
Health and Usage Monitoring Systems (HUMS) have been developed in order to monitor the health condition of helicopter drivetrains, focusing towards early, accurate and on time fault detection with limited false alarms and missed detections. Among other systems, the Main GearBox (MGB) is the heart of the drivetrain, reducing the high input speed generated by the engines, in order to provide the appropriate torque to the main rotors and to other auxiliary systems. HUMS are mounted on helicopters aiming to enhance the operational reliability and to support maintenance decision making, in order to increase the flight safety keeping in the meanwhile the overall maintenance cost low. Currently used HUMS seems to have reached their limits and the need for improvement has been recently highlighted by the post-accident analysis of the helicopter LN-OJF, which crashed in Norway in 2016. The aim of this paper is the application and further extension of recently proposed advanced cyclostationary based signal processing tools for the accurate detection of faults in helicopter gearboxes. The methodologies are tested, evaluated and compared with state of the art methods on datasets captured during experimental tests under various operating conditions on helicopter gearboxes, including a Category A Super Puma SA330 main planetary gearbox.
\end{abstract}

\section{Introduction}

Health and Usage Monitoring Systems (HUMS) conduct helicopter health monitoring by extracting Health Indicators (HI) from acquired vibration data. However, HI's have a wide range of values depending on a plethora of conditions of the helicopter besides their health state (e.g. temperature, speed, torque, etc.), making the implementation of specific thresholds of the HI often unreliable for the health monitoring. Analysis of the time-domain of the vibration data is also non-practical, as MGB of helicopters are complex machinery with many sources. Analysis of the frequency-domain provides a more direct way to monitor the health condition of its components by tracking the amplitude at specific frequencies related to specific components. Signal processing techniques are usually applied in this scenario in

\footnotetext{
*e-mail: konstantinos.gryllias@kuleuven.be
} 
order to detect modulations related to the fault that may be hidden in the spectrum $[1,2]$. One of the most common approaches in bearing fault detection is the Envelope Analysis, where the signal is demodulated in order to detect the lower cyclic modulations of the bearing. This method is sensitive to the noise, so it is commonly used alongside with a band pass filter around the band of frequencies excited by the impulses of the bearing fault. The selection of the band pass filter can be made mainly by two general approaches: exploitation of engineering knowledge and experience; or through band selection tools. The band selection tools provide the advantage of (semi-)automated selection without requiring expertise and/or trial-and-error of band-pass filtering. The most widely used method is the Fast Kurtogram, which maximises the value of Spectral Kurtosis of a series of filters. However, there are other known phenomena that generate high Kurtosis values, resulting in the selection of incorrect bands [3]. In the frames of the analysis of vibration data of a MGB with damaged bearing, Zhou et. al. [1] concluded that the Kurtogram was ineffective by itself, and the damage was only diagnosable after application of a self-adaptive noise cancellation filter. The last decade, the interest in the development and use of cyclostationary based tools, such as the Cyclic Spectral Correlation (CSC) and Cyclic Spectral Coherence (CSCoh) for condition monitoring, has been increased as they achieve high performance in revealing hidden periodicities in their bi-variable representations. Direct analysis of these results is difficult to perform, unless the analyst has extensive prior experience. On the other hand, the integration of the spectral frequency variable results in an one dimensional envelope spectrum, the Enhanced Envelope Spectrum (EES), detailing the cyclic modulations present in the signal. Furthermore, when the integration is performed on a specific narrow band instead of the full band, only the signature with carriers inside the integrated band are kept in the spectrum, nominated as the Improved Envelope Spectrum (IES). This method achieves robust results in extracting hidden modulations in the spectrum, and results in a classical Envelope Spectrum of easy analysis $[4,5]$. Recently, the IESFOgram (Improved Envelope Spectrum via Feature Optimization -gram) has been proposed as an automated band selection tool for application on the CSC and CSCoh bi-variable maps, leading to robust results in the field of bearing diagnostics [6]. The key objective of this paper is to evaluate the performance of the IESFOgram and compare it with state of the art methods by applying it on real vibration data acquired from a MGB of a Super Puma under various levels of damage on the planet bearing, operating under different speed and load conditions.

\section{Filter band selection tools}

The band selection tools follow the same concept. A series of filters is applied to the signal following a $1 / 3$ binary tree filter bank structure. For each filtered iteration, a specific feature is extracted, and the filter band corresponding to the maximum feature is selected as the optimal band. In this paper, several band selection tools are applied, and each maximizes a specific feature. The feature corresponding to each different selection tools are: Fast Kurtogram Spectral Kurtosis of the signal [7], Infogram (time-domain) - Spectral Negentropy of the Squared Envelope[8], Infogram (frequency-domain) - Spectral Negentropy of the Squared Envelope Spectrum[8], L2/L1 norm - Sparsity measure (L2/L1) of the signal [10, 11], Gini Index - Sparsity measure (Gini) of the signal [12].

\subsection{IESFOgram}

Rolling element bearing signals can be described as second-order cyclostationary signals, due to the slippage of elements, presenting a periodic autocorrelation of period $\mathrm{T}$ :

$$
R_{2 x}(\tau, t)=\mathbb{E}\left\{x(t) x(t-\tau)^{*}\right\}=R_{2 x}(\tau, t+T)
$$


where $\mathrm{x}(\mathrm{t})$ is the time signal, $\mathrm{E}$ is the ensemble average and $\tau$ is the time-lag. Cyclostationary based tools, such as Cyclic Spectral Correlation (CSC) and Coherence (CSCoh) have received an ever increasing interest due to their ability in extracting hidden modulations. CSC estimates the correlation between a modulating frequency (the cyclic frequency $\alpha$ ) and a carrier frequency (the spectral frequency $f$ ) presented in a bi-variable map [4]:

$$
\operatorname{CSC}(\alpha, f)=\lim _{T \rightarrow \infty} T^{-1} \mathbb{E}\left\{\mathscr{F}[x(t)] \mathscr{F}[x(t+\tau)]^{*}\right\}
$$

The Cyclic Spectral Coherence (CSCoh) is the whitened version of the CSC:

$$
\operatorname{CSCoh}(\alpha, f)=\frac{\operatorname{CSC}(\alpha, f)}{\sqrt{\operatorname{CSC}(0, f) \operatorname{CSC}(0, f+\alpha)}}
$$

where $\mathscr{F}(x(t))$ is the Fourier transform of the signal $x(t)$. The integration of the bi-variable maps over a band $[F 1, F 2]$ of the spectral frequency $f$ leads to the Improved Envelope Spectrum (IES) and to the Enhanced Envelope Spectrum (EES) $(F 1=0, F 2=F s / 2, F s$ is the sampling frequency) which are equivalent to a demodulation spectrum:

$$
\operatorname{IES}(\alpha)=\frac{1}{F_{2}-F_{1}} \int_{F_{1}}^{F_{2}}|\operatorname{CSCoh}(\alpha, f)| d f
$$

Recently the IESFOgram has been proposed as a tool is order to automatically select the optimal integration band, similarly to the Kurtogram [9]. A series of integrating iterations for different combinations of bandwidth and centre frequency are performed following a $1 / 3$ binary tree. A diagnostic feature $D F$, equal to the sum of the amplitude of $\mathrm{k}=3$ harmonics of the bearing fault frequency $f_{\text {fault }}$ normalized by the surrounding noise level (estimated at a band $\left[f_{\text {fault }}-/+1 / 4 f\right.$ shaf $\left.t\right]$ ), is estimated at each band, as described by Eq.:

$$
D F(c f, b w)=\sum_{k=1}^{N} \frac{I E S_{c f, b w}\left(k \times \alpha_{f a u l t}\right)}{\frac{1}{2 f_{b}}\left[\int_{k f_{\text {fault }}-f_{b}}^{k f_{\text {full }}+f_{b}} I E S_{c f, b w}(\alpha) d \alpha-I E S_{c f, b w}\left(k \times a l p h a_{f a u l t}\right)\right]}
$$

The highest feature value corresponds to the optimal integration band as described in Figure 1.

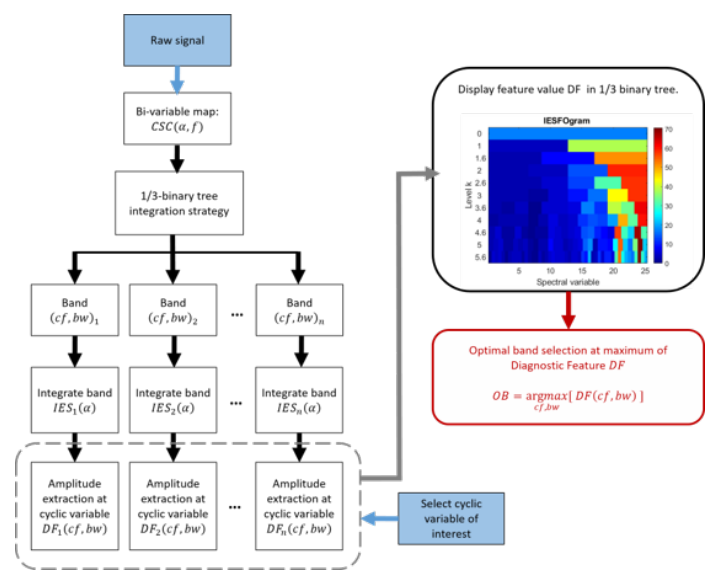

Figure 1. Schematic description of the IESFOgram procedure 


\section{Experimental Setup}

A Category A Super Puma SA330 main Gearbox (MGB) has been used in order to investigate the accuracy of planetary bearing defect diagnosis methods. The measurements have been acquired on a dedicated test rig, presented in Figure 2, in the frames of a project entitled "MGH - Helicopter main gearbox health" sponsored by the European Aviation Safety Agency (EASA) implemented in 2012. The MGB has several stages of reduction, parallel and planetary. Bearing defects have been seeded in the outer race of the planetary bearing at the second epicyclic module. More precisely, the outer race faults have been seeded on the inner bore of the planet gear, which is the outer race of the bearing. The planet gears are driven by the 2 nd epicyclic sun gear and the ring gear.

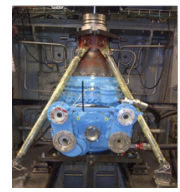

(b)

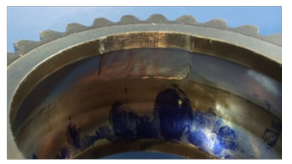

Figure 2. (a) Tested Main Gearbox, (b) Major fault seeded on the planet outer race.

\section{Results}

For each case, different number of accelerometers were used but here are presented only the results based on the signal that is captured by the closest accelerometer to the damage source and that is not corrupted or truncated.

\subsection{Case under $23.000 \mathrm{rpm}$ and $1760 \mathrm{~kW}$ operating conditions}

The vibrations signals analysed in this first case were captured under $23.000 \mathrm{rpm}$ shaft speed and $1760 \mathrm{~kW}$. The signals were also measured under three health condition cases of the outer race of the planet bearing of the second epicyclical stage: healthy, minor fault, and major fault. The minor fault is approximately $10 \mathrm{~mm}$ wide, and the major fault is approximately $30 \mathrm{~mm}$. Based on the kinematics of the main gearbox, the cyclic frequency of interest BPFO is calculated equal to $97.4 \mathrm{~Hz}$. The identification of this component and its harmonics results in the diagnosis of the outer race damage. The SES and EES were also extracted and analysed, however no peaks of the BPFO were detected in any of the cases (due to lack of space the figures have been omitted). The Fast Kurtogram for each health condition, seen in Figure 3, was used to select the band for filtering that results in the SES (based on Fast Kurtogram) presented in Figure 4. The harmonics of the BPFO cannot be detected with confidence above the noise level, as can be seen in the spectra. Similar results are obtained from the Envelope Spectra based on the Infogram from time-domain, Infogram from frequency-domain, L1/L2 binary tree and Gini Index binary tree, that are shown in Figure 5, Figure 6, Figure 7 and Figure 8 respectively. On the other hand, the IESFOgram for the same signals, presented in Figure 9, and its corresponding IES, presented in Figure 10, allows for the detection of the peaks at the BPFO's harmonics well above the noise level for the damaged cases, and thus provides a robust diagnosis. 

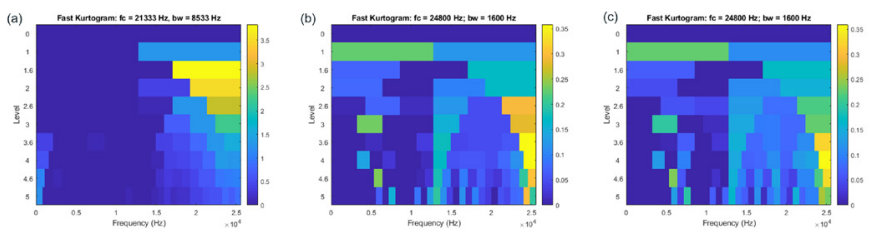

Figure 3. Fast Kurtogram of: (a) healthy case, (b) minor fault case, and (c) major fault case.
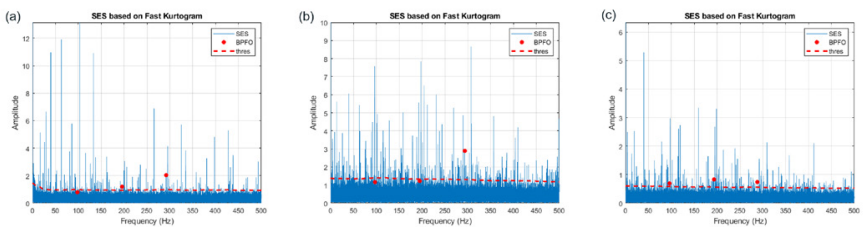

Figure 4. SES based on FK of: (a) healthy case, (b) minor fault case, and (c) major fault case.
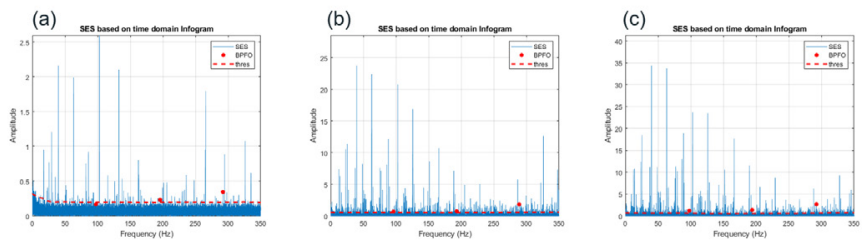

Figure 5. SES based on TD Infogram of: (a) healthy case, (b) minor fault case, and (c) major fault case.
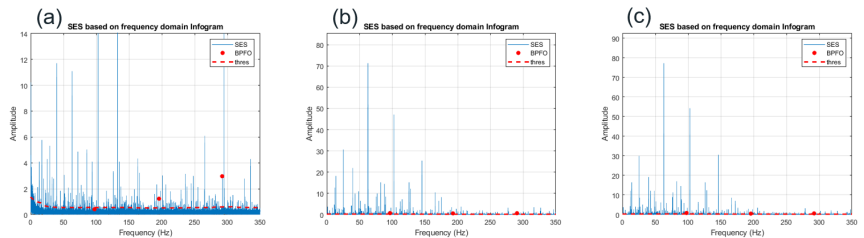

Figure 6. SES based on FD Infogram of: (a) healthy case, (b) minor fault case, and (c) major fault case.

\subsection{Case under $16.000 \mathrm{rpm}$ and $180 \mathrm{~kW}$ operating conditions}

On the second test case, the operating conditions were lower than on the first case, at 16.000 rpm rotation speed and $180 \mathrm{~kW}$. The minor fault has a length, width and depth of $5 \mathrm{~mm}, 3 \mathrm{~mm}$, and $1.5 \mathrm{~mm}$ respectively. On the other hand, the major fault has in turn a length, width and depth of $8 \mathrm{~mm}, 6 \mathrm{~mm}$, and $2.5 \mathrm{~mm}$. Under these speed conditions, the BPFO is estimated equal to $68.5 \mathrm{~Hz}$. The peaks at the BPFO harmonics are not detectable for any health condition at the SES, the EES, the SES based on the Fast Kurtogram, Infogram, L1/L2 and Gini Index, as seen in Figure 11. The IES based on the IESFOgram identifies the first harmonic of the BPFO well above the noise level, however the peak at the BPFO is unexpectedly detected in the healthy case, as demonstrated in Figure 12. The amplitude increases with the severity level, which can provide a diagnosis of increasing damage, but the detection of the BPFO 

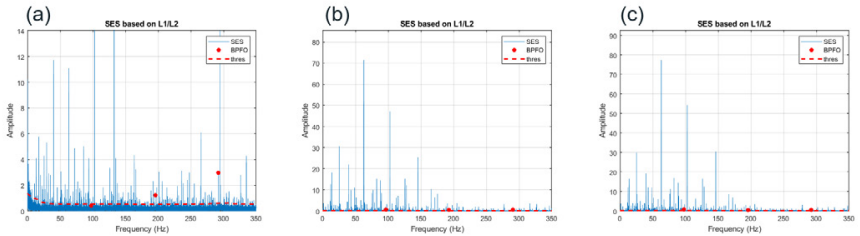

Figure 7. SES based on L1/L2 of: (a) healthy case, (b) minor fault case, and (c) major fault case.
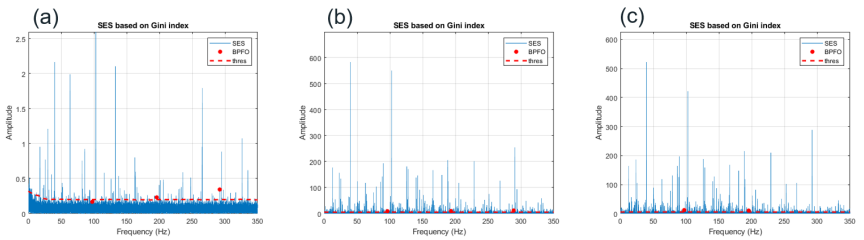

Figure 8. SES based on TD Infogram of: (a) healthy case, (b) minor fault case, and (c) major fault case.
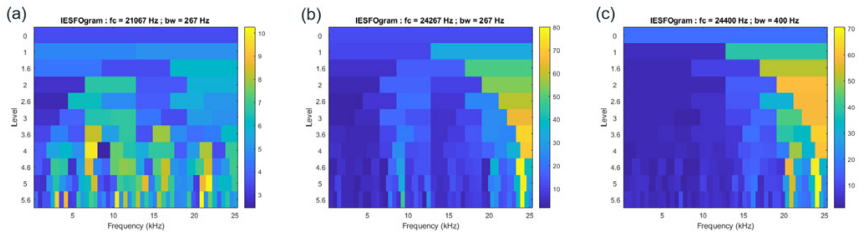

Figure 9. IESFOgram of: (a) healthy case, (b) minor fault case, and (c) major fault case.
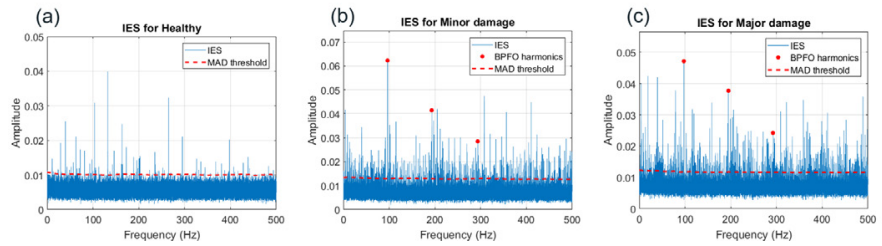

Figure 10. IES based on IESFOgram of: (a) healthy case, (b) minor fault case, and (c) major fault case.

at the healthy case does not provide full confidence in the diagnosis. The peak at the 68.5 $\mathrm{Hz}$ in the healthy case can appear due to: a) accidental damage of the outer race during the bearing mounting on the setup, b) the presence of the ball pass frequency which may appear as a result of the varying stiffness caused by the balls in contact, or c) other component of the drivetrain.

\subsection{Case under $23.000 \mathrm{rpm}$ and $1300 \mathrm{~kW}$ operating conditions}

The last case was only captured for two health conditions: healthy and major damage on the outer race of the bearing. Data was captured under $23.000 \mathrm{rpm}$ and $1300 \mathrm{~kW}$ operating conditions on both situations. In this case, the peaks at the $97.4 \mathrm{~Hz}$ are more easily extracted 

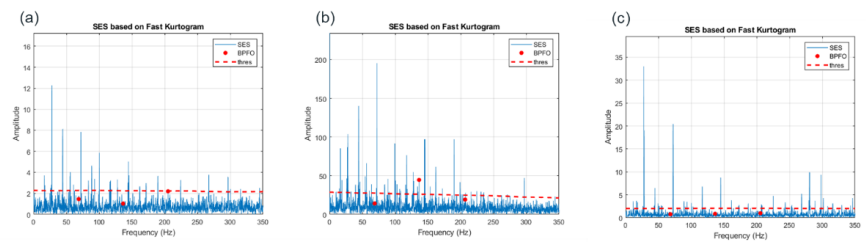

Figure 11. SES based on FK of: (a) healthy case, (b) minor fault case, and (c) major fault case.
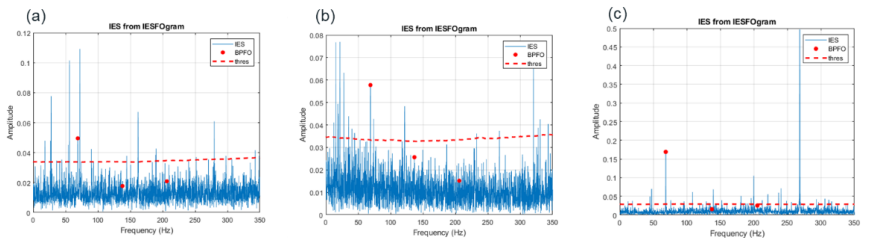

Figure 12. IES based on IESFOgram of: (a) healthy case, (b) minor fault case, and (c) major fault case.

when compared to the previous cases, as the BPFO can be detected not only on the IES based on the IESFOgram, as seen in Figure 13, but also on the classical SES and EES, and SES based on the time-domain Infogram, and Gini Index. The exceptions are the SES based on the Fast Kurtogram, frequency-domain Infogram, and L1/L2, where the BPFO harmonics are not detectable at the major fault spectrum.
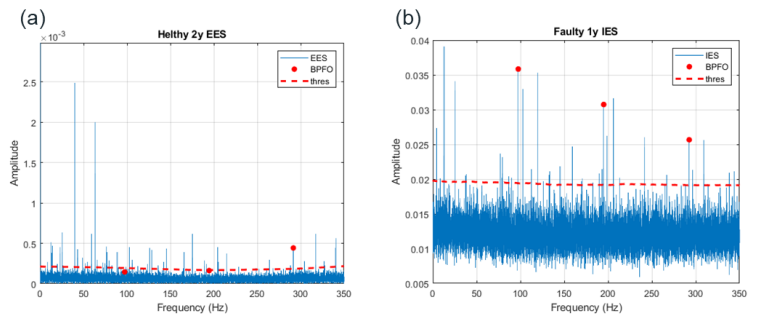

Figure 13. IES based on IESFOgram of: (a) healthy case, and (b) major fault case.

\subsection{Comparison of methodologies}

The results of the methodologies' comparison are briefly presented in Figure 14 for the all analysed cases. The "NO" marks the NO fault detection while the "YES" highlights the fault detection. The green colour corresponds to the correct result while the red colour corresponds to an error. The Fast Kurtogram, Infogram (frequency-domain) and L1/L2 based SES show low performance, either because the signal of interest is well masked by other components, or because an incorrect filtering band is selected. Moreover the diagnosis by the classical SES, EES, Infogram (time-domain), Gini Index analysis is successful for only one of the three cases. The integration of the CSCoh using the band selected from the IESFOgram results in spectra where the BPFO is clearly identified in all the damaged cases, however it is also extracted on the healthy signal of one of the cases. Based on the table of results in 
Figure 14 it can be concluded that the IES estimated based on the IESFOgram achieves the best results compared to the other 3 state of the art methodologies. On the other hand, one false alarm is present and the authors are now working on this issue in order to clarify the reason of appearance and the improvement of the general methodology targeting towards a robust diagnostic method with a limited rate of false alarms and missed detections.

\section{Conclusions}

\begin{tabular}{|c|c|c|c|c|c|c|c|c|c|}
\hline $\begin{array}{c}\text { Operating } \\
\text { conditions }\end{array}$ & Damage & SES & EES & IES & $\begin{array}{c}\text { Fast } \\
\text { Kurtogram }\end{array}$ & $\begin{array}{c}\text { Infogram } \\
\text { (time) }\end{array}$ & $\begin{array}{c}\text { Infogram } \\
\text { (frequency) }\end{array}$ & L1/L2 & Gini Index \\
\hline \multirow{3}{*}{$\begin{array}{l}\text { Speed } 23.000 \mathrm{rpm} \\
\text { Load } 1760 \mathrm{~kW}\end{array}$} & Healthy & NO & No & No & No & NO & No & NO & NO \\
\hline & \begin{tabular}{|c|}
$\begin{array}{c}\text { Minor } \\
\text { damage }\end{array}$ \\
\end{tabular} & NO & No & YES & No & No & No & No & NO \\
\hline & \begin{tabular}{|c|}
$\begin{array}{c}\text { Major } \\
\text { damage }\end{array}$ \\
\end{tabular} & NO & No & YES & NO & No & NO & NO & NO \\
\hline \multirow{3}{*}{$\begin{array}{l}\text { Speed } 16.000 \mathrm{rpm} \\
\text { Load } 180 \mathrm{~kW}\end{array}$} & Healthy & NO & NO & YES & NO & NO & NO & NO & NO \\
\hline & \begin{tabular}{|c|} 
Minor \\
damage
\end{tabular} & NO & No & YES & NO & NO & NO & NO & NO \\
\hline & $\begin{array}{c}\text { Major } \\
\text { damage }\end{array}$ & NO & No & YES & NO & NO & NO & NO & NO \\
\hline \multirow{2}{*}{$\begin{array}{l}\text { Speed } 23.000 \mathrm{rpm} \\
\text { Load } 1300 \mathrm{~kW}\end{array}$} & Healthy & NO & NO & NO & No & NO & No & NO & NO \\
\hline & \begin{tabular}{|c|}
$\begin{array}{c}\text { Minor } \\
\text { damage }\end{array}$ \\
\end{tabular} & YES & YES & YES & No & YES & No & NO & YES \\
\hline
\end{tabular}

Figure 14. Detection of the BPFO for all cases for different signal processing methods.

In this paper, the Improved Envelope Spectrum based on the IESFOgram is used for bearing diagnostics of helicopter's main gearbox. The methodology is applied on signals captured on a Category A Super Puma SA330 helicopter main gearbox transmission, for various speed conditions, load conditions and severity level of damage on a rolling element bearing. The method is evaluated and compared against a plethora of Envelope Spectra based on different band selection tools: Fast Kurtogram, Infogram (time and frequency), L1/L2, and Gini Index. The proposed methodology identifies correctly the fault in all cases presenting only one false alarm, the reasoning of which is currently under investigation.

\section{References}

[1] L. Zhou, F. Duan, M. Corsar, F. Elasha, D. Mba, Applied Acoustics 147, 4-14 (2019)

[2] R. Randall, N. Sawalhi, Shock and Vibrations 5, 451-461 (2011)

[3] W. A. Smith, Z. Fan, Z. Peng, H. Li, R. Randall, Mechanical Systems and Signal Processing 75, 371-394 (2016)

[4] R. Randall, J. Antoni, J. Chobsaard, Mechanical Systems and Signal Processing 15, 945$962(2001)$

[5] D. Abboud, S. Baudin, J. Antoni, D. Remond, M. Eltabach, O, Sauvage, Mechanical Systems and Signal Processing 75, 280-300 (2016)

[6] A. Mauricio, J. Qi, K. Gryllias, Proceedings of ASME TurboExpo (2018)

[7] J. Antoni, Mechanical Systems and Signal Processing 21, 108-124 (2007)

[8] J. Antoni, Mechanical Systems and Signal Processing 74, 73-94 (2016)

[9] A. Mauricio, W. Smith, R. Randall, J. Antoni, K. Gryllias, Proceedings of ISMA (2018)

[10] M. Wang, Z. Mo, H. Fu, H yu, Q. Miao, Special section on Advances in Prognostics and System Health Management IEEE Access (2019)

[11] D. Wang, Y. Zhao, C. Yi, K. Tsui, J. Lin, Mechanical Systems and Signal Processing 101, 292-308 (2018)

[12] Y. Miao, M. Zhao, J. Lin, Measurement Science and Technology 28, 12-25 (2017) 